\title{
Stronger Environmental Adaption of Abundant than Rare Microbes in Wetland Soils from the Qinghai- Tibet Plateau
}

\section{Wenjie Wan}

Chinese Academy of Sciences

\section{Geoffrey Michael Gadd}

University of Dundee

\section{Yuyi Yang}

Chinese Academy of Sciences

\section{Wenke Yuan}

Chinese Academy of Sciences

\section{Jidong Gu}

HKU: University of Hong Kong

\section{Wenzhi Liu ( $\square$ liuwz@wbgcas.cn )}

Chinese Academy of Sciences Fujian Institute of Research on the Structure of Matter https://orcid.org/0000-0002-7149-9529

\section{Research}

Keywords: biogeographic pattern, community assembly, environmental thresholds, phylogenetic signals, Qinghai-Tibet Plateau wetlands, rare versus abundant

Posted Date: October 14th, 2020

DOl: https://doi.org/10.21203/rs.3.rs-89424/v1

License: (c) (1) This work is licensed under a Creative Commons Attribution 4.0 International License. Read Full License 


\section{Abstract}

Background: Disentangling the biogeographic patterns of rare and abundant microbial sub-communities is essential in order to understand the generation and maintenance of microbial diversity with respect to the functions they provide. However, little is known about ecological assembly processes and environmental adaptation of rare and abundant microbial sub-communities across large spatial-scale wetlands. Using Illumina sequencing, we investigated the taxonomic and phylogenetic $\beta$-diversity of rare and abundant bacterial and fungal sub-communities in Qinghai-Tibet Plateau wetland soils. Additionally, we determined environmental breadths and phylogenetic signals of ecological preferences of rare and abundant microbial sub-communities, and investigated community assembly processes of microbial taxa.

Results: We found that both taxonomic and phylogenetic similarities of rare and abundant microbial subcommunities attenuated with geographical distance. Based on threshold indicator taxa analysis and Blomberg's $K$ statistic, abundant microbial taxa exhibited broader environmental thresholds and stronger phylogenetic signals for ecological traits than rare microbial taxa. The strong correlations between community compositional dissimilarity and phylogenetic distance of rare microbial sub-communities also revealed that rare taxa may be more sensitive to environmental changes. In addition, the rare microbial sub-communities exhibited closer phylogenetic clustering compared with abundant microbial sub-communities. The null model analysis revealed that dispersal limitation belonging to stochastic process dominated ecological assembly of abundant bacterial sub-community, and rare and abundant fungal sub-communities; variable selection belonging to deterministic process governed community assembly of rare bacterial taxa. Neutral model analysis and variation partitioning analysis further confirmed that abundant microbial sub-communities were less environmentally constrained. Soil ammonia nitrogen was the crucial factor in mediating the balance between stochasticity and determinism of both rare and abundant microbial sub-communities, as reflected by distinct differences in stochastic process with higher ammonia nitrogen content.

Conclusions: Abundant microbial sub-communities may have better environmental adaptation potential and are less dispersed by environmental changes compared with rare microbial sub-communities. Our findings extend knowledge of the adaptation of rare and abundant microbial taxa to ongoing environmental change and could facilitate prediction of biodiversity loss caused by global climate change and increasing human activity in wetlands of the Qinghai-Tibet Plateau.

\section{Background}

Wetlands, regarded as the kidneys of the Earth, are important ecosystems for flora and fauna biodiversity, providing food, regulating climate, and purifying water [1, 2]. However, global wetlands are facing many ecological problems (e.g., biodiversity loss, reclamation, and water pollution) due to climate change and anthropogenic disturbance [3-5]. Therefore, microbial community in wetlands might also be subject to diversity loss and as a consequence the ecological function of biogeochemical processes will be 
affected. Microorganisms in wetland soils are one of the largest world reservoirs of biodiversity and drive numerous ecological processes in terrestrial ecosystems [6-8]. Specifically, bacteria and fungi are responsible for the turnover and cycling of important elements, including carbon, nitrogen, and phosphorus [9-11]. Microbial diversity is closely correlated with multiple ecosystem functions [6, 10], therefore understanding microbial diversity might be beneficial for evaluating the health of wetland ecosystems.

Deciphering the fundamental mechanisms for generating and maintaining microbial diversity is a core objective in community ecology, and some interesting patterns have been discovered. For instance, distance-decay relationships (DDRs) mean that microbial community similarity generally decreases with an increase in geographical distance $[12,13]$. Microbial $\beta$-diversity varies along environmental gradients (e.g., pH and salinity) $[14,15]$. Many ecological theories that attempt to explain diversity-environment interconnections mainly consider species interaction models (e.g., competition and cooperation) and its in-situ resource (e.g., space and nutrient availability) $[13,16,17]$. Heterogeneity in substrate preference and environmental stress adaptations of microorganisms results in differences in microbial growth and biomass yield $[14,15,18]$. This can lead to a skewed abundance distribution in a local microbial community, with relatively few dominant and a large number of rare species (alternatively known as a "rare biosphere") $[17,19,20]$. Previous studies have reported that rare and abundant species often show different distribution patterns and functional traits [21-23]. Therefore, disentangling the biogeography and community assembly of rare and abundant microbial taxa is essential for understanding microbedriven ecosystem processes and functions.

Recent studies have described the biogeography of rare and abundant microbial taxa in various environments $[17,20]$, with both spatial effects and environmental factors influencing soil microbial diversity $[17,20,24]$. For instance, local physicochemical properties have greater effects on both rare and abundant bacterial $\beta$-diversity compared to geospatial factors [20]. However, most studies investigate the biogeography of rare and abundant bacterial communities in agricultural soils [17, 22, 24, 25], and little is known about rare and abundant bacterial and fungal taxa in natural wetlands.

Environmental filtering is an important determinant in shaping species distribution patterns and affecting abundance [26-28]. The relative abundance of a rare or abundant taxon is the result of a tradeoff between its growth and death rates [29]. Rare and abundant microbial taxa show diverse responses to environmental change [22, 25]. Environmental thresholds of arbuscular mycorrhizal fungi in European grassland are estimated using the accumulated values of change points of all the species in a given microbial community [30]. Procurable environmental thresholds rarely integrate the abundance, occurrence, and directionality of microbial responses at the species level, and little research is based on standardized phylogenetic and molecular evolutionary analysis of natural sites on a large spatial scale $[17,31]$. Additionally, the responses of microbes to environmental change exhibit phylogenetic conservatism, and in this case microbes are not distributed randomly across the tree of life [32, 33]. For example, ectomycorrhizal fungal of Craterellus show strong conservatism of positive response to nitrogen deposition, while Cortinarius, Tricholoma, Piloderma, and Suillus exhibit strong conservatism of 
consistently negative responses to nitrogen deposition [34]. Soil pH and salinity are regarded as crucial determinants in shaping terrestrial bacterial biogeographic patterns $[28,35,36]$, and the response traits of $\mathrm{pH}$ and salinity preference are found to be relatively deeply conserved [32]. Therefore, understanding the phylogenetic patterns of microbial response traits provides predictions for microbial biogeography and their responses to environmental change, and for changes in biodiversity-driven ecosystem multifunctioning $[6,17,37,38]$. However, phylogenetic distributions and response thresholds of both bacterial and fungal communities to ongoing environmental change, especially abundant and rare taxa, have not been evaluated in wetlands of high elevation geographic regions.

Ecological community assembly, regarded as a significant ecological issue, estimates the relative contributions of stochastic processes (i.e., dispersal limitation and homogenizing dispersal) and deterministic processes (i.e., variable selection and homogeneous selection) to microbial communities $[13,39-41]$. Both stochastic and deterministic processes determine microbial communities and are considered to be obligatory in coupling microbial community structure with the ecosystem functions they supply $[40,42]$. Abundant microbial taxa are limited by dispersion more than rare taxa in agricultural soils $[17,21]$ and in inland freshwater ecosystems across China [43]. By contrast, the dispersal of rare bacterial taxa is more limited than that of abundant bacterial taxa in three subtropical bays of China [44]. The balance between stochastic and deterministic processes is regulated by environmental factors $[13,45$, 46]. For instance, the divergence in soil pH and salinity could change the relative contributions of different ecological assembly processes in shaping bacterial communities $[8,21,36,46]$. Moreover, extreme pH even results in a deterministic community assembly of soil bacteria [46]. However, whether similar environmental variables mediating the dominance of stochasticity and determinism in community assembly of rare and abundant microbes in wetlands remains unclear.

The 33 wetlands in the Qinghai-Tibetan Plateau, with altitudes ranging from 2547 to $4745 \mathrm{~m}$, were chosen as our study areas. The mean annual temperature and mean annual precipitation of these wetlands are $-4.49-17.62^{\circ} \mathrm{C}$ and $89-1038 \mathrm{~mm}$, respectively, and detailed terrain properties are described (Additional file 1: Table S1). These wetlands have been largely protected from human activities. However, some factors including climate change might engender some unknown impacts on these wetlands. This situation caught our interest to predict and evaluate the responses of wetland ecosystems to environmental change, and in order to understand protection of wetlands by mitigating the impact of climate change in the future. In the present study, we aimed to (i) assess the potential environmental thresholds and phylogenetic distributions of rare and abundant bacteria and fungi across diverse environmental gradients in wetlands across Qinghai-Tibetan Plateau, and (ii) reveal the major environmental variables affecting the assembly of rare and abundant microbial sub-communities. In view of the low competition potential and growth rate of rare taxa $[47,48]$, we hypothesized that rare microbial taxa would present relatively narrow environmental thresholds and relatively weak phylogenetic signals for traits compared with abundant microbial taxa. In addition, the ecological assembly processes dominating rare and abundant microbial sub-communities would be affected by different environmental variables. To achieve our goals and validate our hypothesis, we employed amplicon sequencing datasets of soil bacteria and fungi along with 14 environmental factors. 


\section{Results}

\section{General distribution patterns of rare and abundant sub- communities}

After quality filtering and reads control, a total of 1,041,535 and 775,601 high-quality sequences were clustered into 4,443 OTUs for bacteria and 1,977 OTUs for fungi. As expected, rare bacteria comprised $66.4 \%$ of the total bacterial richness, but their total relative abundance accounted for only $13.2 \%$ of the entire bacterial community. Conversely, a quite low proportion of $3.91 \%$ OTUs was identified as abundant bacteria, which occupied $47.9 \%$ of the entire bacterial community. Similarly, $61.9 \%$ and $7.4 \%$ OTUs were identified as rare and abundant fungi, and their relative abundances accounted for $5.9 \%$ and $76.5 \%$ of the total fungal community. Abundance-occupancy relationships demonstrated that both rare bacteria and fungi possessed stronger positive correlations than corresponding abundant bacteria and fungi (Fig. 1A; Fig. 1B). $97.1 \%$ abundant bacteria and $54.4 \%$ abundant fungi occurred in more than $50 \%$ of the soil samples, while only $43.2 \%$ rare bacteria and $0.7 \%$ rare fungi existed in more than $50 \%$ of the soil samples. These results suggested that abundant bacteria and fungi presented relatively higher occupancies compared with corresponding rare bacteria and fungi. Across all the samples, the rare bacterial subcommunity was dominated by Proteobacteria (27.8\%) and Chloroflexi (15.5\%), while the abundant bacterial sub-community was dominated by Proteobacteria (55.7\%) and Bacteroidetes (13.3\%) (Fig. 1C). In contrast, Ascomycota (45.2\%) and unclassified fungi (31.6\%) dominated in the rare fungal subcommunity, whereas Ascomycota (34.2\%) and Basidiomycota (32.3\%) were the dominant phyla in the abundant fungal sub-community (Fig. 1D). These results indicate that the rare and abundant bacteria and fungi showed distinct distribution patterns in the wetlands of the Qinghai-Tibet Plateau.

We evaluated both taxonomic and phylogenetic patterns for both rare and abundant microbial subcommunities in the Qinghai-Tibet Plateau wetlands (Fig. 2). Although the DDRs of community similaritygeographical distance (Fig. 2A) and phylogenetic similarity-geographical distance (Fig. 2B) were significant $(p<0.05$ and $p<0.001)$, the fitness values were relatively low $\left(\mathrm{R}^{2}<0.1\right)$, demonstrating weak decays of taxonomic and phylogenetic similarities with geographical distance. Significantly higher taxonomic diversity represented by community distance was found in the rare bacterial sub-community compared with the abundant bacterial sub-community (Wilcoxon $p<0.05$ ), while there was no remarkable difference in taxonomic diversity between rare and abundant fungal sub-communities (Wilcoxon $p>0.05$ ) (Fig. 2C). Similarly, noticeably higher phylogenetic distances for both rare bacterial and fungal subcommunities were higher than those of the corresponding abundant bacterial and fungal subcommunities (Fig. 2D). As expected, the mean values of SES.MNTD were noticeably higher for abundant rather than for rare microbial sub-communities (Additional file 1: Figure S2). Additionally, significantly positive correlations between compositional dissimilarity and phylogenetic distance were observed in both rare and abundant bacterial and fungal sub-communities, and the correlations in rare bacterial and fungal sub-communities were much stronger than those for the corresponding abundant subcommunities (Fig. 3). This might imply that the phylogenies of rare and abundant microbial sub- 
communities exhibited distinct sensitivities to environmental changes. We further found that environmental variables determined more variations in both abundant bacterial and fungal subcommunities than for corresponding rare bacterial and fungal sub-communities based on redundancy analysis (Additional file 1: Figure S3). This suggests that rare bacterial and fungal sub-communities might be more sensitive to environmental change, which could lead to species extinction of some rare ones.

\section{Environmental responses of rare and abundant sub- communities}

Differing correlations between environmental factors and relative abundances of the top 20 rare and abundant microbes were observed, and specific environmental factors determined the relative abundance of specific microbes (Additional file 1: Figure S4 and S5). For instance, longitude significantly affected the relative abundance of OUT_1088 belonging to the Basidiomycota. Environmental threshold analysis was used to explore the responses of rare and abundant microbial sub-communities to each of the environmental variables based on calculations of $z+$ and z- (Additional file 1: Figure S6-S8).

Interestingly, the abundant bacterial sub-community showed a broader range of environmental thresholds compared with the rare bacterial sub-community for almost all the variables except for electrical conductivity (EC) (Fig. 4A). Similarly, the abundant fungal sub-community had a wider range of environmental thresholds than the rare fungal sub-community, excluding $\mathrm{pH}$ (Fig. 4B). The strength of the relationship between environmental preferences and microbial phylogeny was also calculated to determine whether ecological traits could be predictive in terms of phylogenetic diversity (Fig. 4C and 4D). Blomberg's $K$ statistic signified that the abundant bacterial and fungal sub-communities presented stronger phylogenetic signals for all environmental variables compared with the corresponding rare bacterial and fungal sub-communities (Fig. 4C and 4D). These results suggested that within the abundant microbial sub-communities, closely correlated taxa showed similar ecological preferences to the environmental factors. Phylum-level taxonomy confirmed such observations (Additional file 1: Figure S9 and S10). For example, the abundance taxa of Acidobacteria, Actinobacteria, Bacteroidetes, Firmicutes, and Proteobacteria exhibited stronger phylogenetic signals for all 14 environmental variables (Additional file 1: Figure S9). The abundant taxa of Ascomycota (64.3\%), Basidiomycota (100\%), and Chytridiomycota $(71.4 \%)$ presented stronger phylogenetic signals for more than $60 \%$ of the 14 environmental variables (Additional file 1: Figure S10).

\section{Ecological community assembly processes in rare and abundant microbial sub-communities}

The relative contributions of ecological processes differed in microbial sub-communities based on null model analysis (Additional file 1: Figure S11). Dispersal limitation belonging to stochastic processes dominated in the abundant bacterial sub-community, rare and abundant fungal sub-communities, with corresponding relative contributions of $72.5 \%, 75.8 \%$, and $90.5 \%$, respectively. Variable selection (69.9\%) 
belonging to deterministic processes governed the rare bacterial sub-community assembly. Homogenizing dispersal and homogeneous selection, belonging to homogenizing process, had little impact on both rare and abundant bacterial and fungal sub-community assembly.

Mantel tests revealed that change in soil ammonia nitrogen $\left(\mathrm{NH}_{4}{ }^{+}-\mathrm{N}\right)$ was significantly correlated with $\beta N T I$ of rare bacterial $(r=-0.117 ; p<0.001)$, abundant bacterial $(r=-0.072 ; p<0.05)$, rare fungal $(r=$ $-0.084 ; p<0.01)$, and abundant fungal $(r=-0.139 ; p<0.001)$ sub-communities (Table 1$)$. This suggested that ammonia nitrogen might be the optimal predictor of assembly processes in both rare and abundant bacterial and fungal sub-communities. In addition, pairwise comparisons of $\beta N T I$ values for rare and abundant microbial sub-communities were noticeably and negatively correlated with changes in soil ammonia nitrogen (Additional file 1: Figure S12). This indicated that an increasing divergence in ammonia nitrogen resulted in a shift from variable selection to stochasticity in the assembly of both rare and abundant bacterial and fungal sub-communities. To further investigate the relationship between ammonia nitrogen and phylogenetic turnover $(\beta \mathrm{NTI})$, soils were separated into subgroups based on ammonia content. With increasing soil ammonia nitrogen, the relative contributions of stochasticity increased in the rare bacterial sub-community and decreased in the abundant bacterial sub-community, first decreased and then increased in the rare fungal sub-community, and first increased and then declined in the abundant fungal sub-community (Additional file 1: Figure S13). 
Table 1

Mantel tests of environmental variables against $\beta N T I$ of rare and abundant microbial sub-communities. Asterisks denote significance $(*, p<0.05 ; * \star, p<0.01 ; * \star \star, p<0.001)$

\begin{tabular}{|c|c|c|c|c|}
\hline \multirow[t]{2}{*}{ Property } & \multicolumn{2}{|c|}{ Bacterial community } & \multicolumn{2}{|c|}{ Fungal community } \\
\hline & Rare & Abundant & Rare & Abundant \\
\hline Lon & $0.155^{\star \star \star}$ & -0.058 & $0.090 \star \star$ & $0.119 \star \star \star$ \\
\hline Lat & 0.005 & $-0.080 * \star$ & 0.015 & $0.124^{\star \star \star}$ \\
\hline Alt & -0.005 & 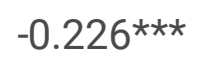 & $0.090 \star \star$ & 0.043 \\
\hline MAT & -0.035 & $-0.183^{\star \star \star}$ & $0.173^{\star \star \star}$ & 0.007 \\
\hline MAP & 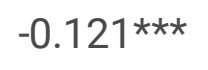 & $-0.100 \star \star \star$ & $0.081^{\star \star}$ & 0.050 \\
\hline PR & -0.012 & -0.038 & -0.021 & 0.038 \\
\hline PC & 0.045 & -0.006 & 0.016 & 0.036 \\
\hline Tem & $-0.120 * \star \star$ & 0.008 & 0.012 & -0.018 \\
\hline $\mathrm{pH}$ & 0.006 & $-0.101 \star \star \star$ & 0.020 & -0.038 \\
\hline EC & 0.041 & 0.017 & $0.063^{*}$ & 0.049 \\
\hline Moisture & -0.013 & 0.047 & -0.005 & -0.017 \\
\hline TC & $0.088 * \star$ & $0.061^{*}$ & 0.060 & $0.106^{\star \star \star}$ \\
\hline TP & $0.089 * \star$ & $0.082^{\star \star}$ & 0.042 & 0.015 \\
\hline $\mathrm{NH}_{4}^{+}-\mathrm{N}$ & 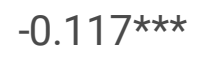 & $-0.072^{\star}$ & $-0.084^{\star \star}$ & $-0.139 * \star \star$ \\
\hline
\end{tabular}

To make the community assembly of stochasticity more comparable, variation partitioning analysis and neutral analysis were also employed. The ratios of sorting/dispersal limitation were higher in rare bacterial and fungal sub-communities than that in the corresponding abundant bacterial and fungal subcommunities based on variation partitioning analysis (Fig. 5A) and null model analysis (Fig. 5B). These results suggested that the abundant microbial sub-communities were less environmentally constrained. Neutral model analysis further validated this finding with relatively higher $m$ values in the abundant microbial sub-communities (Fig. 5C).

\section{Discussion}

\section{Broader environmental adaptations of abundant microbial taxa}


Environmental filtering governs microbial communities and in turn affects ecosystem function [27, 40, 49]. Numerous studies have investigated the effects of environmental variables on the compositions of rare and abundant microbial communities, despite their adaptations to the environment $[20,24,50]$. We have attempted to provide insights into the responses of rare and abundant microbial sub-communities to ongoing environmental change. Here, the environmental adaptations of microbial sub-communities were clarified in two contexts: environmental breadth based on TITAN analysis [17, 51] and the levels of phylogenetic signals to ecological preference based on Blomberg's $K$ statistic [37, 52]. Environmental breadth can reflect species niche breadth, and phylogenetic signals could provide predictions for microbial evolutionary adaptation in response to environmental change [32, 53].

Firstly, we found that abundant bacterial and fungal sub-communities showed broader response thresholds to environmental factors than the corresponding rare bacterial and fungal sub-communities. These results are in line with the finding that the abundant fungal sub-community exhibits higher environmental thresholds than rare fungal taxa in agricultural soils of China [17]. This phenomenon might be due to the relatively easier access and broader nutrient utilization potential of abundant microbial taxa compared with rare taxa $[18,19]$. Indeed, the abundant microbial taxa were omnipresent compared with rare microbial taxa across wetlands in Qinghai-Tibet Plateau, which is in accordance with previous findings [17, 20,44], and could support the perspective described above. Rare bacterial and fungal taxa were not distributed evenly, and most taxa occurred only in a few soils. This might be attributed to the low growth rate and competition potential of rare microbial taxa, thus constraining in their environmental breadth $[48,54]$. Our results emphasized that both abundant bacterial and fungal sub-communities had higher niche breadths, which reflected their adaptations to broader ranges of environmental gradients. Environmental threshold analysis based on TITAN has been reported in some biodiversity-related studies [17, 30,55]. For instance, ectomycorrhizal fungal diversity in North American Pinaceae forests determines large effects on ectomycorrhizal fungi-associated biogeochemical cycles [55]. The results of environmental breadths of microbes based on environmental threshold analysis are attractive, but are also controversially discussed regarding implications to the real field situation.

Therefore, confirmatory experiments are needed when applying such statistical results to environmental policy. Nevertheless, our results based on sequencing datasets provide statistical hint for the potentially broader environmental breadths of abundant bacterial and fungal taxa compared with the corresponding rare bacterial and fungal taxa in wetlands across the Qinghai-Tibet Plateau. These findings are, to our knowledge, novel and have not been reported before.

Secondly, we observed that abundant bacterial and fungal taxa exhibited stronger phylogenetic signals for environmental preference compared with rare microbial taxa based on Blomberg's $K$ statistic. The results are consistent with findings reported for Chinese agricultural soils [17] and grassland and forest soils across North America [56]. Such findings might reveal that closely correlated species exhibit more similar ecological preferences across environmental gradients within the abundant microbial subcommunities. Prior studies have clarified that functional traits based on the ecological preferences of an organism depend on species evolutionary history $[57,58]$. For instance, traits of bacteria in four forest ecosystems are to a higher degree constrained by evolutionary history than environmental heterogeneity 
(e.g., temperature, precipitation, and dominant vegetation) [59]. Additionally, the response traits of salinity and $\mathrm{pH}$ preferences for environmental microorganisms are found to be deeply phylogenetically conserved [32], which might be related to the decisive roles of salinity and $\mathrm{pH}$ in shaping microbial biogeography [8, $17,35,36]$. In the present study, abundant microbial taxa exhibiting stronger phylogenetic signals for ecological preferences might reveal that abundant taxa possessed more phylogenetic niche conservatism with respect to the evolutionary history of environmental adaptation [60]. Interestingly, tighter connections

(Fig. 3, larger $\mathrm{R}^{2}$ values) between compositional dissimilarity and phylogenetic distance were observed in rare rather than abundant microbial sub-communities. This phenomenon might be mainly attributed to the phylogenies of abundant microbial sub-communities being less sensitive to ongoing environmental change [20]. The potential to maintain a community's phylogeny could reveal the capacity of the community to preserve the ecological niche $[61,62]$. Therefore, the decoupling between community composition and phylogenetic distance indicates that abundant bacteria and fungi are relatively better in maintaining ecological niches than the corresponding rare bacteria and fungi. These findings might explain why abundant taxa have broader environmental breadths and distinct biogeographic patterns compared with rare microbial taxa. In summary, the results of environmental breadth and phylogenetic signal analyses show that rare and abundant bacterial and fungal sub-communities possessed distinct adaptations to various environmental conditions in wetlands across the Qinghai-Tibet Plateau.

\section{Different assembly processes dominating rare and abundant sub-communities}

Deciphering ecological processes underlying community assembly is a mainstream problem in microbial ecology [8,63-65]. Microbial community composition and functions are regarded as being governed by different ecological mechanisms and therefore it is essential to quantify the relative contributions of stochastic and deterministic processes to microbial community assembly $[42,66,67]$. Here, the stochastic process (i.e., dispersal limitation) dominated in the abundant bacterial sub-community, and the rare and abundant fungal sub-communities; the deterministic process (i.e., variable selection) was dominant in the rare bacterial sub-community. These results agree with previous findings that abundant bacterial and fungal sub-communities are dominated by dispersal limitation [17, 21], while differing from the reports of rare bacterial and/or fungal sub-communities being governed by homogeneous selection $[17,21]$ and stochasticity $[20,44]$. These inconsistencies might be due to differences in habitat characteristics and geography [8]. In addition, some discrepancies might also be due to the differences in cell size and lifestyle of bacteria and fungi [68]. Soil bacteria have much smaller cell sizes compared with filamentous fungi [69]. Cell size has often been regarded as an important factor in affecting the dispersal potential of organisms $[68,70]$. Due to the effects of cell size on bacteria and fungi, dispersal abilities and metabolic activities may affect the adequacy of stochasticity or determinism for explaining their community composition $[42,53,68]$. Despite this, our findings still support previous studies reporting that abundant microbial taxa are more limited by dispersion than rare taxa in agricultural soils $[17,21]$ and inland freshwater ecosystems [43]. The variation partitioning analysis and neutral model analysis further validate that abundant taxa are less environment constrained in our study and other studies [17, 71]. Additionally, the phylogenetic clustering represented by the SES.MNTD value was more distinct in rare 
microbial sub-communities in our work as in other studies [17, 72]. A previous study has reported that environmental filtering could mediate phylogenetic clustering in a bacterial community [73].

To understand community assembly mechanisms in microbial ecology, it is critical to disentangle the environmental factors influencing the relative contributions of stochastic and deterministic processes [40, $67,74]$. Previously published literatures have revealed that organic matter, $\mathrm{pH}$, available sulfur, and salinity are crucial factors affecting bacterial community assembly processes in soils collected from many different environments [21, 36, 40,46,64]. However, there are limited studies on the environmental variables influencing the relative contributions of stochasticity and determinism that mediate soil fungal community assembly, especially in wetland soils. Based on pairwise community comparison using a null model, we observed that the $\beta N T I$ of both rare and abundant bacterial and fungal sub-communities to be more closely correlated with soil ammonia nitrogen than other factors. This might suggest that soil ammonia nitrogen is the crucial factor adjusting the balance between stochastic and deterministic processes for both rare and abundant microbial sub-communities in the Qinghai-Tibet Plateau wetlands.

The key role of soil ammonia nitrogen in governing both rare and abundant bacterial and fungal community assembly processes might be partially attributed to the functions of nitrogenous nutrients and the physicochemical characteristics of bacteria and fungi [75]. Ammonia nitrogen is an important nutrient for plant and microbial growth [76], and plays a significant role in nitrogen cycling and nitrogencycling-related microbial communities in wetland soils of the Qinghai-Tibet Plateau [77]. Moreover, ammonia nitrogen is closely correlated with soil bulk density, which in turn affects gas diffusion (e.g., oxygen and carbon dioxide) and microbial activity [78]. We therefore conjectured that soil ammonia might affect microbial community assembly via two pathways: (i) directly influencing microbial metabolism and growth, and (ii) indirectly shaping soil bulk density. Considering the coupling of microbial nitrogen cycling with carbon, phosphorus, and sulfur cycling [66, 79], other nutrients (e.g., available phosphorus, organic matter, and available sulfur) might also partially mediate the balance between stochastic and deterministic processes. Future work will investigate more environmental variables in determining community assembly processes. Despite this, we found soil ammonia nitrogen to play a decisive role in governing microbial community assembly in Qinghai-Tibet Plateau wetland soils which is, to our knowledge, unprecedented.

Ultimately, we have constructed a conceptual paradigm to describe the environmental adaptation and ecological community assembly processes of rare and abundant bacterial and fungal sub-communities in wetland soils from the Qinghai-Tibet Plateau (Fig. 6). Abundant bacterial and fungal sub-communities exhibited broader environmental breadths and stronger phylogenetic signals of ecological preference than corresponding rare bacterial and fungal sub-communities. Rare microbial sub-communities exhibited closer phylogenetic clustering than the abundant microbial sub-communities. Deterministic processes dominated in the rare bacterial sub-community; stochastic processes governed abundant bacterial sub-community, and rare and abundant fungal sub-communities. Soil ammonia was the crucial factor in shaping the balance between community assembly processes of rare and abundant microbial sub-communities, showing distinct changes in stochasticity with higher ammonia content. 


\section{Conclusions}

Collectively, we characterized taxonomic and phylogenetic diversity, phylogenetic clustering, environmental breadth, and phylogenetic signal of rare and abundant bacterial and fungal subcommunities along environmental gradients in wetlands from the Qinghai-Tibet Plateau. To our knowledge, our DNA-based datasets and statistical analysis first reveal that abundant bacteria and fungi have better environmental adaptation potentials than corresponding rare one in wetland soils from the Qinghai-Tibet Plateau. Abundant bacterial and fungal sub-communities are less environmentally constrained than corresponding rare bacterial and fungal sub-communities. Soil ammonia nitrogen shows decisive role in mediating the balance between stochastic and deterministic processes in community assemblies of both rare and abundant bacteria and fungi. Based on large-scale sequencing datasets, our present work has highlighted the environmental adaptation of rare and abundant bacterial and fungal sub-communities, and deciphered the environmental factors mediating community assembly processes. Our findings are of significance for understanding the maintenance of microbial diversity, and offer prediction of microbial responses to environmental changes caused by global climate change and human activity in the Qinghai-Tibet Plateau wetlands. Considering the importance of microbial biodiversity for ecosystem functions, confirmatory experiments for the conceptual model should be implemented in various ecosystems in future research.

\section{Methods}

\section{Soil collection and physicochemical properties}

In July 2014, a total of 33 wetlands across Qinghai-Tibetan Plateau (Additional file 1: Figure S1), covering lacustrine, riverine, and palustrine locations, were selected for collection of experimental rhizosphere soils. Detailed sampling information is described in a previous study [80], and the collected soils were stored at $4{ }^{\circ} \mathrm{C}$. The terrain properties of each site including longitude (Lon), latitude (Lat), altitude (Alt), mean annual temperature (MAT), and mean annual precipitation (MAP), plant richness (PR), and plant coverage $(\mathrm{PC})$ were recorded.

Soil physicochemical properties including temperature (Tem), pH, electrical conductivity (EC), moisture (Moi), total carbon (TC), total phosphorus (TP), and ammonia nitrogen ( $\mathrm{NH} 4)$ were measured, as described previously [80].

\section{Molecular analyses}

Soil total DNA from this set of samples was extracted by using a FastDNA Spin Kit for Soil (QBiogene, Carlsbad, CA) after the soil was lyophilized and ground. Extracted DNA was further purified by removing humic substances and potential enzyme inhibitors using a Geneclean Spin Kit (QBiogene, Carlsbad, CA). DNA concentrations were determined using a NanoDrop 2000 Spectrophotometer (Thermo Fisher Scientific, Waltham, MA, USA). All extracted DNA samples were stored at $-80^{\circ} \mathrm{C}$. 
The V3-V4 region of the bacterial 16S rRNA gene was amplified by employing the primers 338F (5'-ACT CCT ACG GGA GGC AGC A-3') and 806R (5'-GGA CTA CHV GGG TWT CTA AT-3') [81]. The fungal internal transcribed spacer (ITS) region was amplified by applying the primers ITS1 (5'-GGA AGT AAA AGT CGT AAC AAG G-3') and ITS2 (5'-GCT GCG TTC TTC ATC GAT GC-3') [82]. Each sample was amplified in triplicate in a $20-\mu \mathrm{L}$ reaction under the following conditions: an initial denaturation at $95^{\circ} \mathrm{C}$ for $3 \mathrm{~min}, 30$ cycles of $95^{\circ} \mathrm{C}$ for $30 \mathrm{~s}, 58^{\circ} \mathrm{C}$ for $30 \mathrm{~s}$, and $72{ }^{\circ} \mathrm{C}$ for $40 \mathrm{~s}$, and then a final extension at $72{ }^{\circ} \mathrm{C}$ for $10 \mathrm{~min}$. The PCR products from each sample were pooled and purified by gel electrophoresis and extracted using an AxyPrep DNA Gel Extraction Kit (Axygen, Hangzhou, China). Sequencing was performed on an Illumina Miseq platform at Novogene Bioinformatics Technology Co., Ltd (Beijing, China). The Miseq raw sequencing data were deposited in the National Center for Biotechnology Information (NCBI, http://www.ncbi.nlm.nih.gov) Short Read Archive (SRA) database under accession number PRJNA657692 for bacteria and PRJNA657715 for fungi.

The raw reads were processed following the pipeline of QIIME2 [83]. To minimize the impact of randomsequencing errors, we removed (i) sequences that did not exactly match primers and barcodes; (ii) sequences with an average quality score < 20; (iii) sequences that contained ambiguous base calls; and (iv) sequences with maximum homopolymers $<10 \mathrm{bp}$. The purified sequences were clustered into operational taxonomic units (OTUs) at a 97\% similarity level, with bacterial taxonomy assessed against the SILVA v132 reference and fungal taxonomy against UNITE v8.0 reference.

\section{Data analysis}

We removed OTUs that contained less than 20 reads to avoid random effects on the identification of rare taxa [17]. Randomly selected subsets of 32,098 reads for bacteria and 24,513 reads for fungi were used to standardize the sequencing effort across samples [17]. The rare and abundant OTUs were defined following recent reports $[17,47]$. Briefly, OTUs with relative abundances below $0.01 \%$ of the total sequences were designated as "rare", those with relative abundances above $0.1 \%$ were designated as "abundant", and the remaining OTUs (0.01-0.1\%) were defined as "intermediate".

To estimate the phylogenetic clustering of rare and abundant taxa, the standardized index of effect size measure of the mean nearest taxon distance (SES.MNTD) was calculated based on the null model using the "ses.mntd" function in the "picante" package of R [84]. To evaluate the pairwise phylogenetic distance between communities, beta mean nearest taxon distance (BMNTD) was calculated using the "comdistnt" function. The distance-decay relationship was determined as the slope of an ordinary least-square between phylogenetic similarity (1-BMNTD) and geographical distance.

The threshold values of rare and abundant taxa in response to each environmental factor described above were measured using threshold indicator taxa analysis in the "TITAN2" package of R [51]. The sums of taxa scores for both bacterial and fungal OTUs were used to determine upper and lower thresholds of difference in the rare and abundant communities based on environmental variables. Additionally, we obtained potential trait information about both rare and abundant microbial taxa. Briefly, the ecological preferences for each OTU were determined by calculating Spearman correlations between 
environmental variables and relative abundances of microbial taxa [56]. The OTUs positively or negatively correlated with salinity were regarded as "salinity-preferred" or "salinity-disliked"; the OTUs positively or negatively correlated with pH were designated as "alkaline-preferred" and "acid-preferred". Subsequently, we applied Blomberg's $K$ statistic to calculate the phylogenetic signals for the environmental preference of rare and abundant taxa $[17,37,56]$. The Blomberg's $K$ statistic is an approach describing phylogenetic signal that compares the observed signal in a trait to the signal under a Brownian motion-based metric of trait evolution on a phylogeny [52]. The $K$ value was calculated applying the "multiPhylosigal" function in the "picante" package of $\mathrm{R}$, where $K$ values higher than 1 imply strong phylogenetic signals and conservatism of traits, and $K$ values closer to 0 denote a convergent or random pattern of evolution [84].

The community assembly processes of rare and abundant microbial communities were evaluated by using variation partitioning analysis, null model, and neutral model [13, 20, 39, 74]. The variation partitioning analysis can distinguish the pure effects of geospatial factors and environmental variables on microbial community composition $[8,20]$. Pure environmental effects without a geospatial component denotes the effect of species sorting (deterministic process), while pure geospatial effects without an environmental variable represents the effect of dispersal limitation [13]. The null model analysis was conducted to calculate the relative contributions of four ecological processes, namely, homogeneous selection, variable selection, dispersal limitation, and homogenizing dispersal [17, 39, 40,46]. Briefly, null model-based Bray-Curtis-based Raup-Crick $\left(\mathrm{RC}_{\text {bray }}\right)$ and $\beta$-nearest taxon index $(\beta \mathrm{NTI})$ were applied to calculate the differences in taxonomic and phylogenetic diversity using the "picante" package of R. If $\beta N T I<-2$ or $\beta N T I>2$, deterministic processes govern the community assembly, with distinctly more (i.e., variable selection; $\beta \mathrm{NTI}>2$ ) and less (i.e., homogeneous selection; $\beta \mathrm{NTI}<-2$ ) phylogenetic turnover than expected. Whereas if $|\beta N T I|<2, R_{\text {bray }}<-0.95$ and $R_{\text {bray }}>0.95$ denote the relative contribution of homogenizing dispersal and dispersal limitation, respectively. The $|\beta N T I|<2$ and $\left|R C_{\text {bray }}\right|<0.95$ were estimated as the influence of "undominated" assembly, namely, no single process drives variations in community structure $[13,40,63]$. The effects of environmental factors on microbial community assembly were evaluated using the Mantel test, where environmental factor dissimilarity was calculated using Euclidean distance matric. The linear regression between change in environmental variable and $\beta N T I$ was generated based on ordinary least-squares. The neutral model was selected to further reveal the contribution of the stochastic process to the rare and abundant microbial community assembly by predicting the interconnection between species abundance distribution and species area [85]. In this model, the migration rate " $m$ " was calculated using the "stats4" and "hmisc" packages of R [13]. Low $m$ value demonstrates that the microbial community is more dispersal limited [86].

\section{Declarations}

\section{Acknowledgements}


We thank all those who donated their time and resources to assist with soil collection. The authors also thank Wenli Chen for providing us a server to handle our Illumina sequencing data.

\section{Funding}

This work was supported by the National Natural Science Foundation of China (32022051), the Youth Innovation Promotion Association of Chinese Academy of Sciences (No. 2017388), and the National Science and Technology Basic Special Project of China (2019FY100603).

\section{Authors' contributions}

YY and WL designed the whole experiment and collected the soil samples. YY, WL, YW, and WW conducted all experiments. WW analyzed the data and wrote the manuscript. YY, WL, GMG, and JG revised the manuscript, and WL submitted the manuscript.

\section{Availability of data and materials}

The Miseq raw sequencing data were deposited in the NCBI Short Read Archive database under accession number PRJNA657692 for bacteria and PRJNA657715 for fungi.

\section{Ethics approval and consent to participate}

Not applicable.

\section{Consent for publication}

Not applicable.

\section{Competing interests}

The authors declare that they have no competing interests.

\section{References}

1. Martins PD, Danczak RE, Roux S, Frank J, Borton MA, Wolfe RA, et al. Viral and metabolic controls on high rates of microbial sulfur and carbon cycling in wetland ecosystems. Microbiome. 2018;138:6.

2. An J, Liu C, Wang Q, Yao M, Rui J, Zhang S, et al. Soil bacterial community structure in Chinese wetlands. Geoderma. 2019;337:290-299. 
3. Gauci V, Matthews E, Dise N, Walter B, Koch D, Granberg G, et al. Sulfur pollution suppression of the wetland methane source in the 20th and 21st centuries. Proc Natl Acad Sci USA. 2004;101:1258312587.

4. Price EPF, Spyreas G, Matthews JW. Wetland compensation and its impacts on $\beta$-diversity. Ecol Appl. 2019;29:e01827.

5. Wang D, Bai J, Wang W, Zhang G, Cui B, Liu X, et al. Comprehensive assessment of soil quality for different wetlands in a Chinese delta. Land Degrad Dev. 2018;29:3783-3794.

6. Wagg C, Bender SF, Widmer F, van der Heijden MG. Soil biodiversity and soil community composition determine ecosystem multifunctinality. Proc Natl Acad Sci USA. 2014;111:5266-5270.

7. Louca S, Polz MF, Mazel F, Albright MBN, Huber JA, O'Connor Ml, et al. Function and functional redundancy in microbial systems. Nat Ecol Evol. 2018;2:936-943.

8. Shi Y, Li Y, Xiang X, Sun R, Yang T, He D, et al. Spatial scale affects the relative role of stochasticity versus determinism in soil bacterial communities in wheat fields across the North China Plain. Microbiome. 2018;6:27.

9. Ge T, Yuan H, Zhu H, Wu X, Nie S, Liu C, et al. Biological carbon assimilation and dynamics in a flooded rice-soil system. Soil Biol Biochem. 2012;48:39-46.

10. Luo G, Rensing C, Chen H, Liu M, Wang M, Guo S, et al. Deciphering the associations between soil microbial diversity and ecosystem multifunctionality driven by long-term fertilization management. Funct Ecol. 2018;32:1103-1116.

11. Wan W, Hao X, Xing Y, Liu S, Zhang X, Li X, et al. Spatial differences in soil microbial diversity caused by pH-driven organic phosphorus mineralization. Land Degrad Dev. 2020a;doi:10.1002/Idr.3734.

12. Martiny JBH, Eisen JA, Penn K, Allison SD, Horner-Devine C. Drivers of bacterial $\beta$-diversity depend on spatial scale. Proc Natl Acad Sci USA. 2011;108:7850-7854.

13. Jiao S, Yang Y, Xu Y, Zhang J, Lu Y. Balance between community assembly processes mediates species coexistence in agricultural soil microbiomes across eastern China. ISME J. 2020;14:202216.

14. Rousk J, Bååth E, Brookes PC, Lauber CL, Lozupone C, Caporaso JG, et al. Soil bacterial and fungal communities across a pH gradient in an arable soil. ISME J. 2010;4:1340-1351.

15. Rath KM, Fierer N, Murphy DV, Rousk J. Linking bacterial community composition to soil salinity along environmental gradients. ISME J. 2019;13:836-846.

16. Ghoul M, Mitri S. The ecology and evolution of microbial competition. Trends Microbiol. 2016;24:833-845.

17. Jiao S, Lu Y. Abundant fungi adapt to broader environmental gradients than rare fungi in agricultural fields. Global Change Biol. 2020a;00:1-15.

18. Zhalnina K, Louie KB, Hao Zhao, Mansoori N, da Rocha UN, Shi S, et al. Dynamic root exudate chemistry and microbial substrate preferences drive patterns in rhizosphere microbial community assembly. Nat Microbiol. 2018;3:470-480. 
19. Jia X, Dini-Andreote F, Falcão Salles J. Community assembly processes of the microbial rare biosphere. Trends Microbiol. 2018;26: 738-747.

20. Ji M, Kong W, Stegen J, Yue L, Wang F, Dong X, et al. Distinct assembly mechanisms underlie similar biogeographical patterns of rare and abundant bacteria in Tibetan Plateau grassland soils. Environ Microbiol. 2020;22:2261-2272.

21. Jiao S, Lu Y. Soil pH and temperature regulate assembly processes of abundant and rare bacterial communities in agricultural ecosystems. Environ Microbiol. 2020b; doi:10.1111/1462-2920.14815.

22. Liang Y, Xiao X, Nuccio EE, Yuan M, Zhang N, Xue K, et al. Differentiation strategies of soil rare and abundant microbial taxa in response to changing climatic regimes. Environ Microbiol. 2020;22:1327-1340.

23. Chen QL, Ding J, Zhu D, Hu HW, Delgado-Baquerizo M, Ma YB, et al. Rare microbial taxa as the major drivers of ecosystem multifunctionality in long-term fertilized soils. Soil Biol Biochem. 2020;doi:10.1016/j.soilbio.2019.107686.

24. Hou J, Wu L, Liu W, Ge Y, Mu T, Zhou T, Li Z, et al. Biogeography and diversity patterns of abundant and rare bacterial communities in rice paddy soils across China. Sci Total Environ. 2020; doi:10.1016/j.scitotenv.2020.139116.

25. Kurm V, Geisen S, Gera Hol WH. A low proportion of rare bacterial taxa responds to abiotic changes compared with dominant taxa. Environ Microbiol. 2019;21:750-758.

26. Kivlin SN, Winston GC, Goulden ML, Treseder KK. Environmental filtering affects soil fungal community composition more than dispersal limitation at regional scales. Fungal Ecol. 2014;12:1425.

27. Bahram M, Hildebrand F, Forslund SK, Anderson JL, Soudzilovskaia NA, Bodegom PM, et al. Structure and function of the global topsoil microbiome. Nature. 2018;560:233-237.

28. Jiao S, Xu Y, Zhang J, Lu Y. Environmental filtering drives distinct continental atlases of soil archaea between dryland and wetland agricultural ecosystems. Microbiome. 2019;7:15.

29. Ratzke C, Denk J, Gore J. Ecological suicide in microbes. Nat Ecol Evol. 2018;2:867-872.

30. Ceulemans T, Van Geel M, Jacquemyn H, Boeraeve M, Plue J, Saar L, et al. Arbuscular mycorrhizal fungi in European grasslands under nutrient pollution. Global Ecol Biogeogr. 2019;00:1-10.

31. van der Linde S, Suz LM, Orme CDL, Cox F, Andreae H, Asi E, et al. Environment and host as largescale controls of ectomycorrhizal fungi. Nature. 2018;558:243-248.

32. Martiny JBH, Jones SE, Lennon JT, Martiny AC. Microbiomes in light of traits: A phylogenetic perspective. Science. 2015;350:aac9323.

33. Isobe K, Allison SD, Khalili B, Martiny AC, Martiny JBH. Phylogenetic conservation of bacterial responses to soil nitrogen addition across continents. Nat Commun. 2019;10:2499.

34. Lilleskov EA, Hobbie EA, Horton TR. Conservation of ectomycorrhizal fungi: exploring the linkages between functional and taxonomic responses to anthropogenic $\mathrm{N}$ deposition. Fungal Ecol. 2011;4:174-183. 
35. Griffiths RI, Thomson BC, James P, Bell T, Bailey M, Whiteley AS. The bacterial biogeography of British soils. Environ Microbiol. 2011;13:1642-1654.

36. Zhang K, Shi Y, Cui X, Yue P, Li K, Liu X, et al. Salinity is a key determinant for soil microbial communities in a desert ecosystem. mSystems. 2020;doi:10.1128/mSystems.00225-18.

37. Goberna M, Verdú M. Predicting microbial traits with phylogenies. ISME J. 2016;10:959-967.

38. Goberna M, Verdú M. Phylogenetic-scale disparities in the soil microbial diversity-ecosystem functioning relationship. ISME J. 2018;12:2152-2162.

39. Stegen JC, Lin XJ, Fredrickson JK, Chen XY, Kennedy DW, Murray CJ, et al. Quantifying community assemble processes and identifying features that impose them. ISME J. 2013;7:2069-2079.

40. Feng Y, Chen R, Stegen JC, Guo Z, Zhang J, Li Z, et al. Two key features influencing community assembly processes at regional scale: Initial state and degree of change in environmental conditions. Mol Ecol. 2018;27:5238-5251.

41. Chen W, Ren K, Isabwe A, Chen H, Liu M, Yang J. Stochastic processes shape microeukaryotic community assembly in a subtropical river across wet and dry seasons. Microbiome. 2019;7:138.

42. Xun W, Li W, Xiong W, Ren Y, Liu Y, Miao Y, et al. Diversity-triggered deterministic bacterial assembly constrains community functions. Nat Commun. 2019;10:3833.

43. Liu L, Yang J, Yu Z, Wilkinson DM. The biogeography of abundant and rare bacterioplankton in the lakes and reservoirs of China. ISME J. 2015;9:2068-2077.

44. Mo Y, Zhang W, Yang J, Lin Y, Yu Z, Lin S. Biogeographic patterns of abundant and rare bacterioplankton in three subtropical bays resulting from selective and neutral processes. ISME J. 2018;12:2198-2210.

45. Logares R, Deutschmann IM, Junger PC, Giner CR, Krabberød AK, Schmidt TSB, et al. Disentangling the mechanisms shaping the surface ocean microbiota. Microbiome. 8:55.

46. Tripathi BM, Stegen JC, Kim M, Dong K, Adams JM, Lee YK. Soil pH mediates the balance between stochastic and deterministic assembly of bacteria. ISME J. 2018;12:1072-1083.

47. Campbell BJ, Yu L, Heidelberg JF, Kirchman DL. Activity of abundant and rare bacteria in a coastal ocean. Proc Natl Acad Sci USA. 2011;108:12776-12781.

48. Jousset A, Bienhold C, Chatzinotas A, Gallien L, Gobet A, Kurm V, et al. Where less may be more: How the rare biosphere pulls ecosystems strings. ISME J. 2017;11:853-862.

49. Li HY, Wang H, Wang HT, Xin PY, Xu XH, Ma Y, et al. The chemodiversity of paddy soil dissolved organic matter correlates with microbial community at continental scales. Microbiome. 2018;6:187.

50. Jiao S, Chen W, Wei G. Biogeography and ecological diversity patterns of rare and abundant bacteria in oil-contaminated soils. Mol Ecol. 2017;26: 5305-5317.

51. Baker ME, King RS. A new method for detecting and interpreting biodiversity and ecological community thresholds. Methods Ecol Evol. 2010;1:25-37.

52. Blomberg SP, Garland T, Ives AR. Testing for phylogenetic signal in comparative data: behavioral traits are more labile. Evolution. 2003;57:717-745. 
53. Gao C, Montoya L, Xu L, Madera M, Hollingsworth J, Purdom E, et al. Fungal community assembly in drought-stressed sorghum shows stochasticity, selection, and universal ecological dynamics. Nat Commun. 2020;11:34.

54. Reveillaud J, Maignien L, Eren AM, Huber JA, Apprill A, Sogin ML, et al. Host-specificity among abundant and rare taxa in the sponge microbiome. ISME J. 2014;8:1198-1209.

55. Steidinger BS, Bhatnagar JM, Vilgalys R, Taylor JW, Qin C, Zhu K, et al. Ectomycorrhizal fungal diversity predicted to substantially decline due to climate changes in North American Pinaceae forests. J Biogeogr. 2020;47:772-782.

56. Oliverio AM, Bradford MA, Fierer N. Identifying the microbial taxa that consistently response to soil warming across time and space. Global Change Biol. 2017;23:2117-2129.

57. Graham CH, Fine PVA. Phylogenetic beta diversity: linking ecological and evolutionary processes across space in time. Ecol Lett. 2008;11:1265-1277.

58. Saladin B, Thuiller W, Graham CH, Lavergne S, Maiorano L, Salamin N. Environment and evolutionary history shape phylogenetic turnover in European tetrapods. Nat Commun. 2019;10:249.

59. Morrissey EM, Mau RL, Hayer M, Allen Liu XJ, Schwartz E, Dijkstra P, et al. Evolutionary history constrains microbial traits across environmental variation. Nat. Ecol. Evol. 2019;3:1064-1069.

60. Bennett JR, Cumming BF, Ginn BK, Smol JP. Broad-scale environmental response and niche conservatism in lacustrine diatom communities. Global Ecol Biogeogr. 2010;19:724-732.

61. Miller ET, Zanne AE, Ricklefs RE. Niche conservatism constrains Australian honeyeater assemblages in stressful environments. Ecol Let. 2013;16:1186-1194.

62. Pyron AR, Costa GC, Patten MA, Burbrink FT. Phylogenetic niche conservatism and the evolutionary basis of ecological speciation. Biol Rev. 2015;90:1248-1262.

63. Stegen JC, Lin X, Konopka AE, Fredrickson JK. Stochastic and deterministic assembly processes in subsurface microbial communities. ISME J. 2012;6:1653-1664.

64. Dini-Andreote F, Stegen JC, van Elsas JD, Salles JF. Disentangling mechanisms that mediate the balance between stochastic and deterministic processes in microbial succession. Proc Natl Acad Sci USA. 2015;112:E1326-E1332.

65. Zhang X, Johnston ER, Liu W, Li L, Han X. Environmental changes affect the assembly of soil bacterial community primarily by mediating stochastic processes. Global Change Biol. 2016;22:198207.

66. Liang Y, Zhang X, Zhou J, Li G. Long-term oil contamination increases deterministic assembly processes in soil microbes. Ecol Appl. 2015;25:1235-1243.

67. Stegen JC, Fredrickson JK, Wilkins MJ, Konopka AE, Nelson WC, Arntzen EV, et al. Groundwatersurface water mixing shifts ecological assembly processes and stimulates organic carbon turnover. Nat Commun. 2016;7:11237.

68. Zinger L, Taberlet P, Schimann H, Bonin A, Boyer F, De Barba M, et al. Body size determines soil community assembly in a tropical forest. Mol Ecol. 2019;28:528-543. 
69. Powell JR, Karunaratne S, Campbell CD, Yao HY, Robinson L, Singh BK. Deterministic process vary during community assembly for ecological dissimilar taxa. Nat Commum. 2015;6:8444.

70. Bailey RI, Molleman F, Vasseur C, Woas S, Prinzing A. Large body size constrains dispersal assembly of communities even across short distances. Sci Rep. 2018;8:10911.

71. Xue Y, Chen H, Yang JR, Liu M, Huang B, Yang J. Distinct patterns and processes of abundant and rare eukaryotic plankton communities following a reservoir cyanobacterial bloom. ISME J. 2018;12:2263-2277.

72. Fan K, Cardona C, Li Y, Shi Y, Xiang X, Shen C, et al. Rhizosphere-associated bacterial network structure and spatial distribution differ significantly from bulk soil in wheat crop fields. Soil Biol Biochem. 2017;113:275-284.

73. Huber P, Metz S, Unrein F, Mayora G, Sarmento H, Devercelli M. Environmental heterogeneity determines the ecological processes that govern bacterial metacommunity assembly in a floodplain river system. ISME J. 2020; doi:10.1038/s41396-020-0723-2.

74. Wan W, Liu S, Li X, Xing Y, Chen W, Huang Q. Dispersal limitation driving phoD-harboring bacterial community assembly: a potential indicator for ecosystem multifunctionality in long-term fertilized soils. Sci Total Environ. 2020c; doi:10.1016/j.scitotenv.2020.141960.

75. Zhong Y, Liu J, Jia X, Shangguan Z, Wang R, Yan W. Microbial community assembly and metabolic function during wheat straw decomposition under different nitrogen fertilization treatments. Biol Fert Soils. 2020;doi:10.1007/s00374-020-01438-z.

76. Blázquez E, Bezerra T, Lafuente J, Gabriel D. Performance, limitations and microbial diversity of a biotrickling filter for the treatment of high loads of ammonia. Chem Eng J. 2017;311:91-99.

77. Ma L, Jiang X, Liu G, Yao L, Liu W, Pan Y, et al. Environmental factors and microbial diversity and abundance jointly regulate soil nitrogen and carbon biogeochemical processes in Tibetan wetlands. Environ Sci Technol. 2020;54:3267-3277.

78. Pan H, Liu H, Liu Y, Zhang Q, Luo Y, Liu X, et al. Understanding the relationships between grazing intensity and the distribution of nitrifying communities in grassland soils. Sci Total Environ. 2018;634:1157-1164.

79. Slate ML, Sullivan BW, Callaway RM. Desiccation and rehydration of mosses greatly increases resource fluxes that alter soil carbon and nitrogen cycling. J Ecol. 2019;107:1767-1778.

80. Yang Y, Liu G, Ye C, Liu W. Bacterial community and climate change implication affected the diversity and abundance of antibiotic resistance genes in wetlands on the Qinghai-Tibetan Plateau. J Hazard Mater. 2019;361:283-293.

81. Wan W, Tan J, Wang Y, Qin Y, He H, Wu H, et al. Responses of the rhizosphere bacterial community in acidic crop soil to $\mathrm{pH}$ : Changes in diversity, composition, interaction, and function. Sci Total Environ. 2020b; doi:10.1016/j.scitotenv.2019.134418.

82. Buée M, Reich M, Murat C, Morin E, Nilsson RH, Uroz S, et al. 2009. 454 Pyrosequencing analyses of forest soils reveal an unexpectedly high fungal diversity. New Phytol. 2009;184:449-456. 
83. Bolyen E, Rideout JR, Dillon MR, Bokulich NA, Abnet CC, Al-Ghalith GA, et al. Reproducible, interactive, scalable and extensible microbiome data science using QIIME 2. Nat Biotechnol. 2019;37:852-857.

84. Kembel SW, Cowan PD, Helmus MR, Cornwell WK, Morlon H, Ackerly DD, et al. Picante: R tools for integrating phylogeines and ecology. Bioinformatics. 2010;26:1463-1464.

85. Zhou J, Ning D. Stochastic community assembly: Does it matter in microbial ecology? Microbiol Mol Biol R;2017;81:e00002-17.

86. Sloan WT, Lunn M, Woodcock S, Head IM, Nee S, Curtis TP. Quantifying the roles of immigration and chance in shaping prokaryotic community structure. Environ Microbiol. 2006;8:732-740.

\section{Figures}
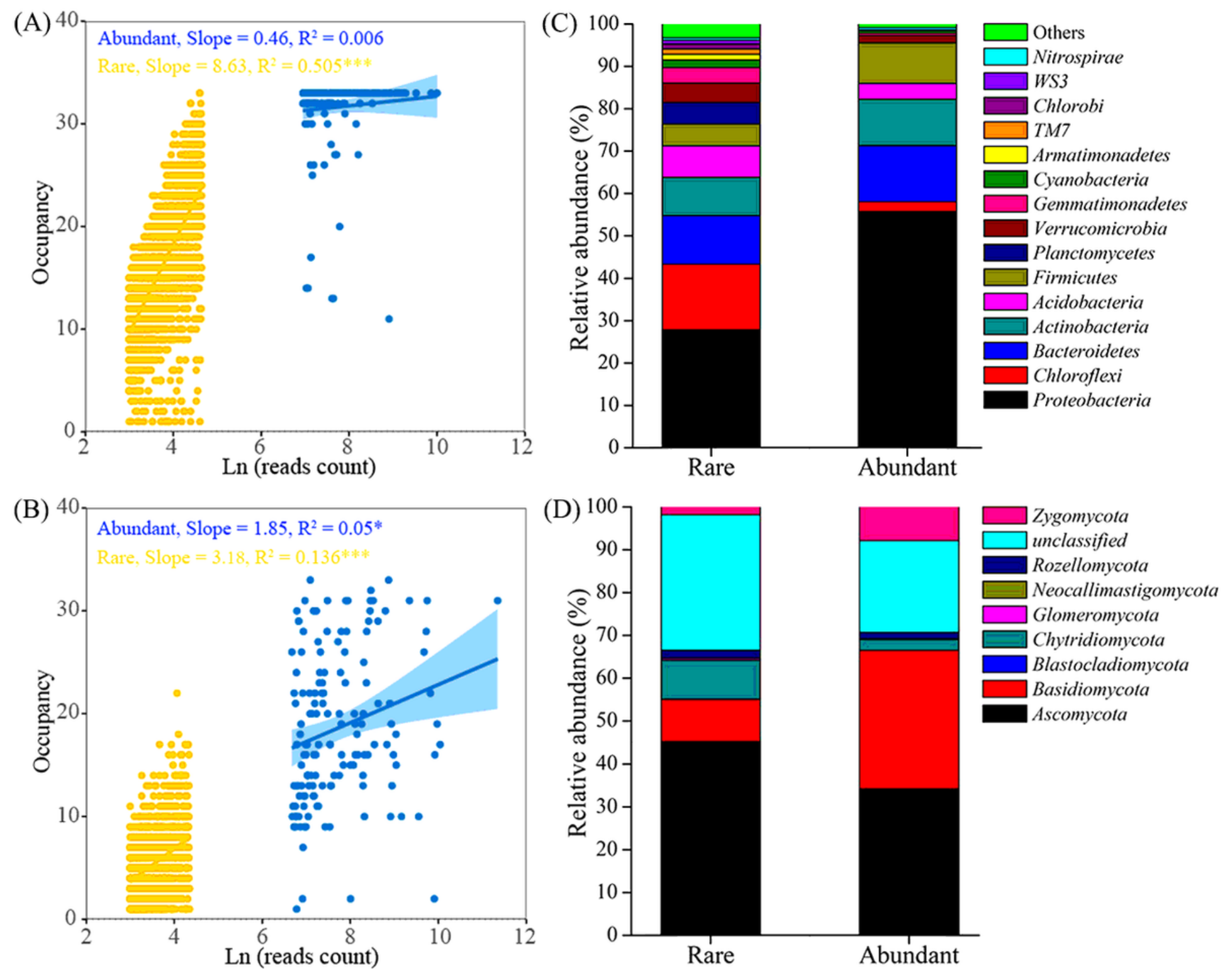

Figure 1 
Abundance-occupancy relationships and taxonomic composition of rare and abundant microbial subcommunities. (A) and (B) show the abundance-occupancy relationships of rare and abundant OTUs. (C) and (D) reflect the taxonomic composition of rare and abundant microbes at the phylum level. Asterisks denote significance $(*, p<0.05 ; * \star *, p<0.001)$
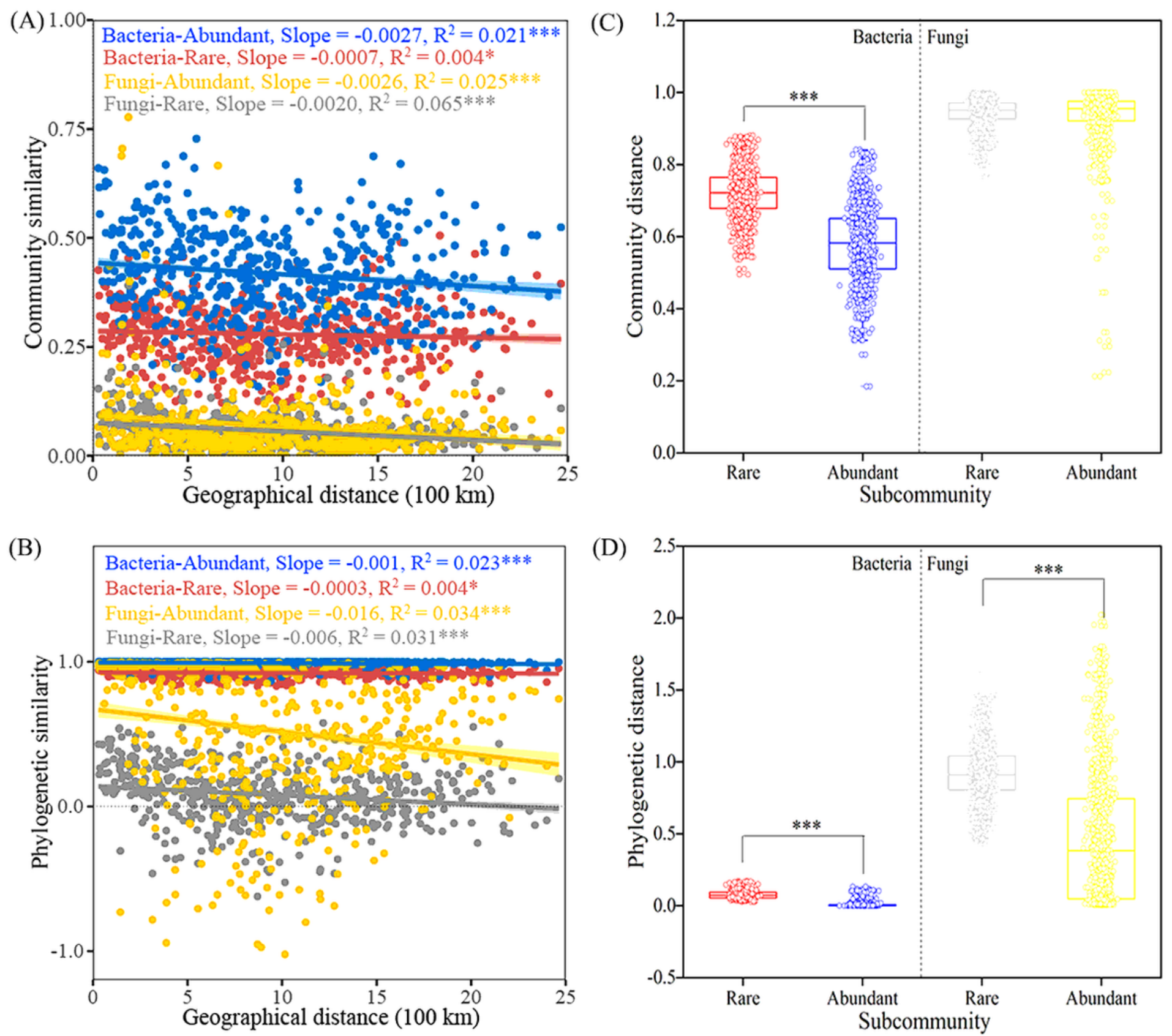

Figure 2

Patterns of rare and abundant microbial $\beta$-diversity. (A) distance-decay curves of community similarity for rare and abundant microbial sub-communities. (B) community distance between rare and abundant subcommunities. (C) distance-decay curves of phylogenetic similarity for rare and abundant microbial subcommunities. (D) phylogenetic distance between rare and abundant sub-communities. Asterisks denote significance $(*, p<0.05 ; * \star \star, p<0.001)$ 


\section{Bacteria-rare}

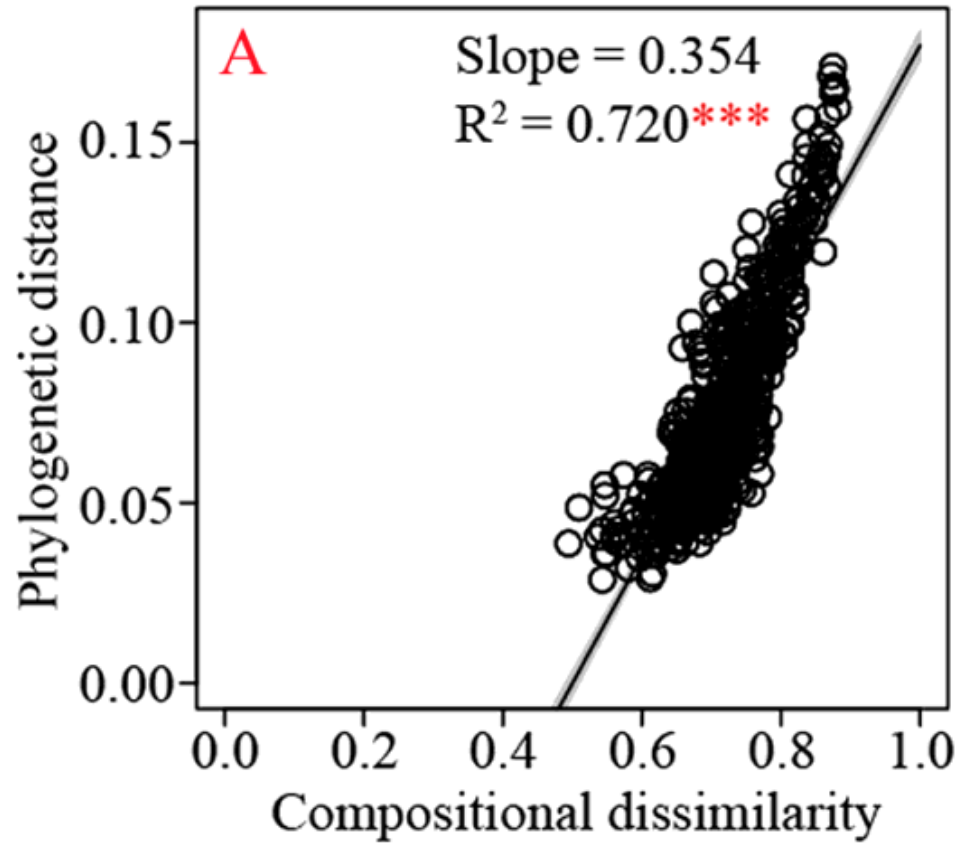

Bacteria-abundant

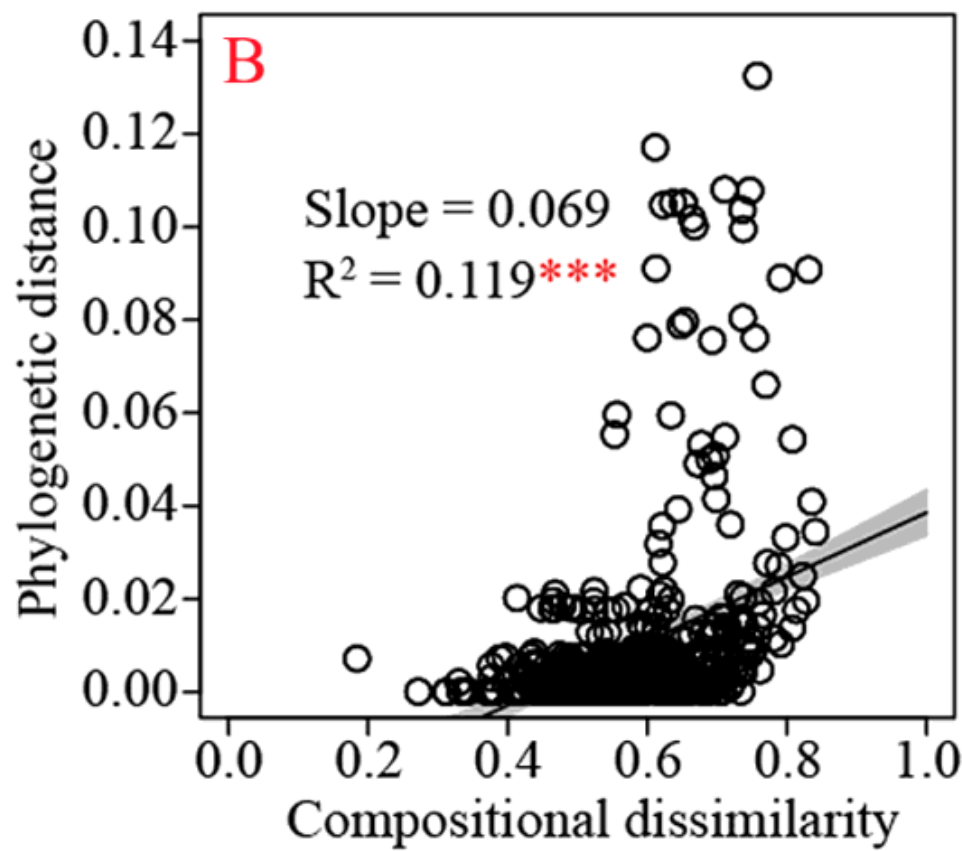

Fungi-rare

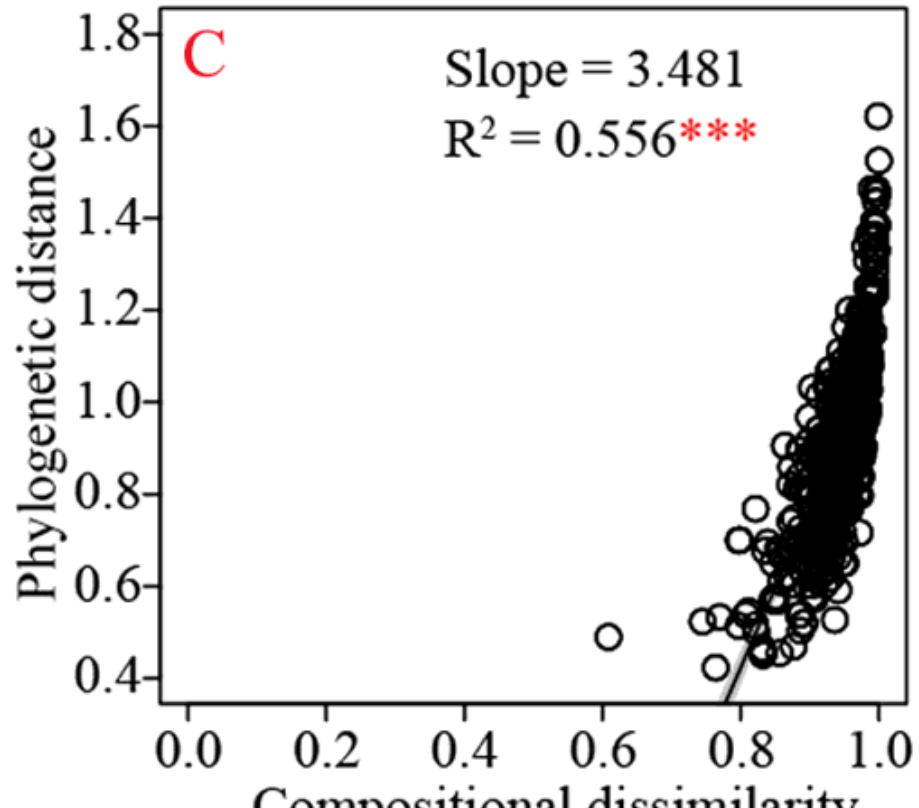

Compositional dissimilarity

Fungi-abundant

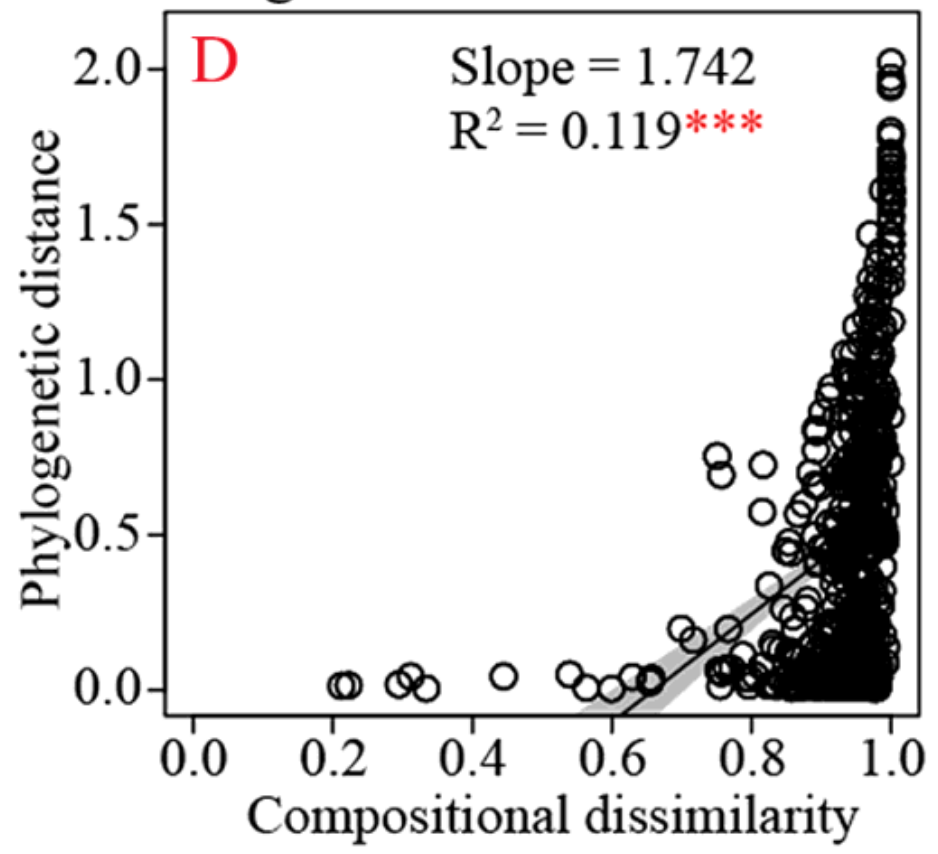

Figure 3

Relationships between phylogenetic distance and compositional dissimilarity of rare $(A)$ and abundant (B) bacterial communities, rare (C) and abundant (D) fungal communities. Asterisks represent significance level $(* \star *, p<0.001)$ 

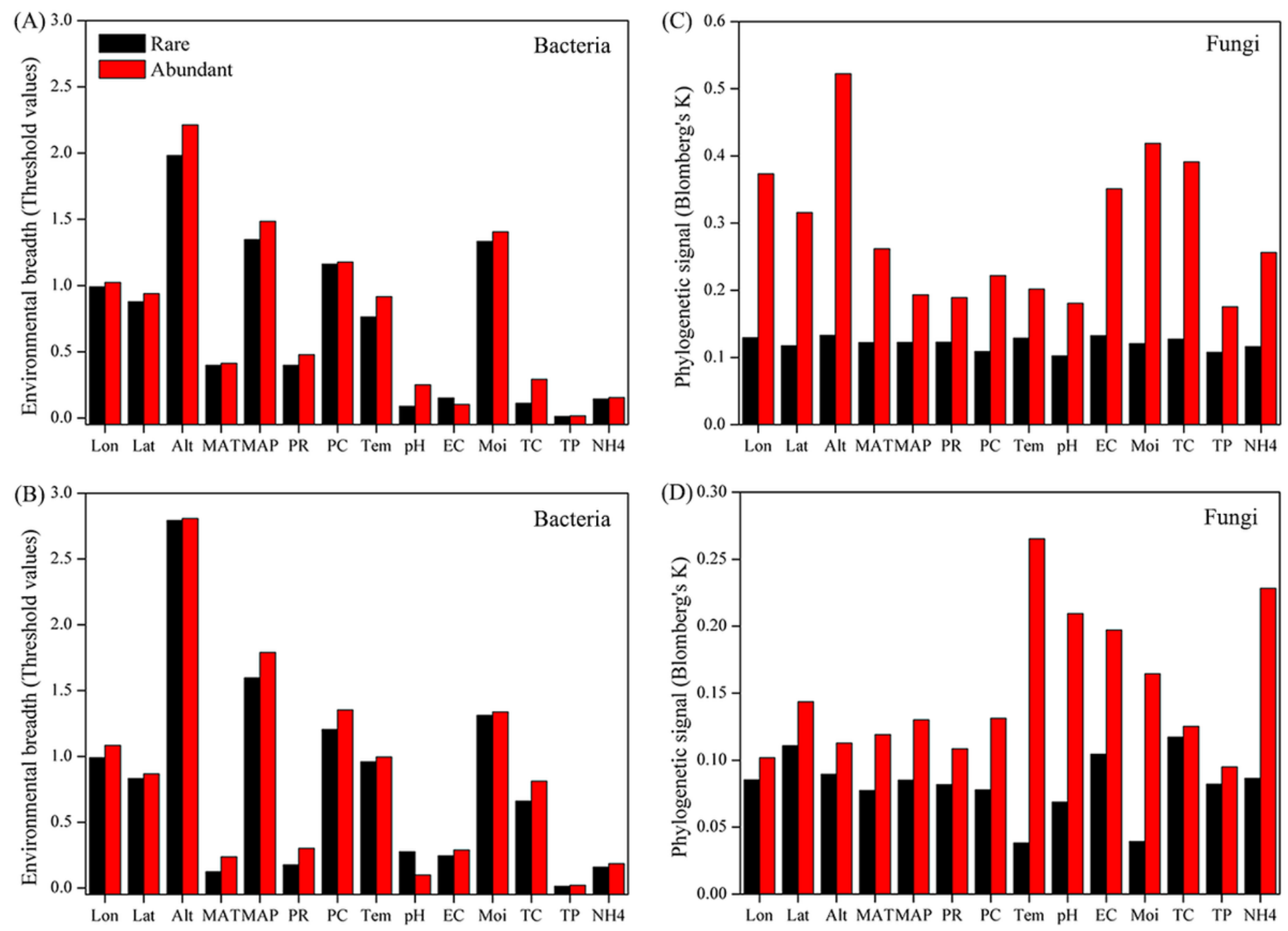

Figure 4

Environmental adaptation of rare and abundant microbial taxa in wetland soils. Environmental breadths of bacteria (A) and fungi (B) evaluated by the threshold values of rare and abundant taxa in response to environmental factors were measured using TITAN. The threshold values in the figures were standardized using $\log 10$ (original threshold value +1 ). Phylogenetic signals of bacteria (C) and fungi (D) reflecting the trait conservatism for environmental preferences of the rare and abundant sub-communities were determined applying Blomberg's K statistic. Lon, Lat, Alt, MAT, MAP PR, PC, Tem, EC, Moi, TC, TP, and NH4 represent longitude, latitude, altitude, mean annual temperature, mean annual precipitation, plant richness, plant coverage, temperature, electrical conductivity, moisture, total carbon, total phosphorus, and ammonia nitrogen, respectively. 

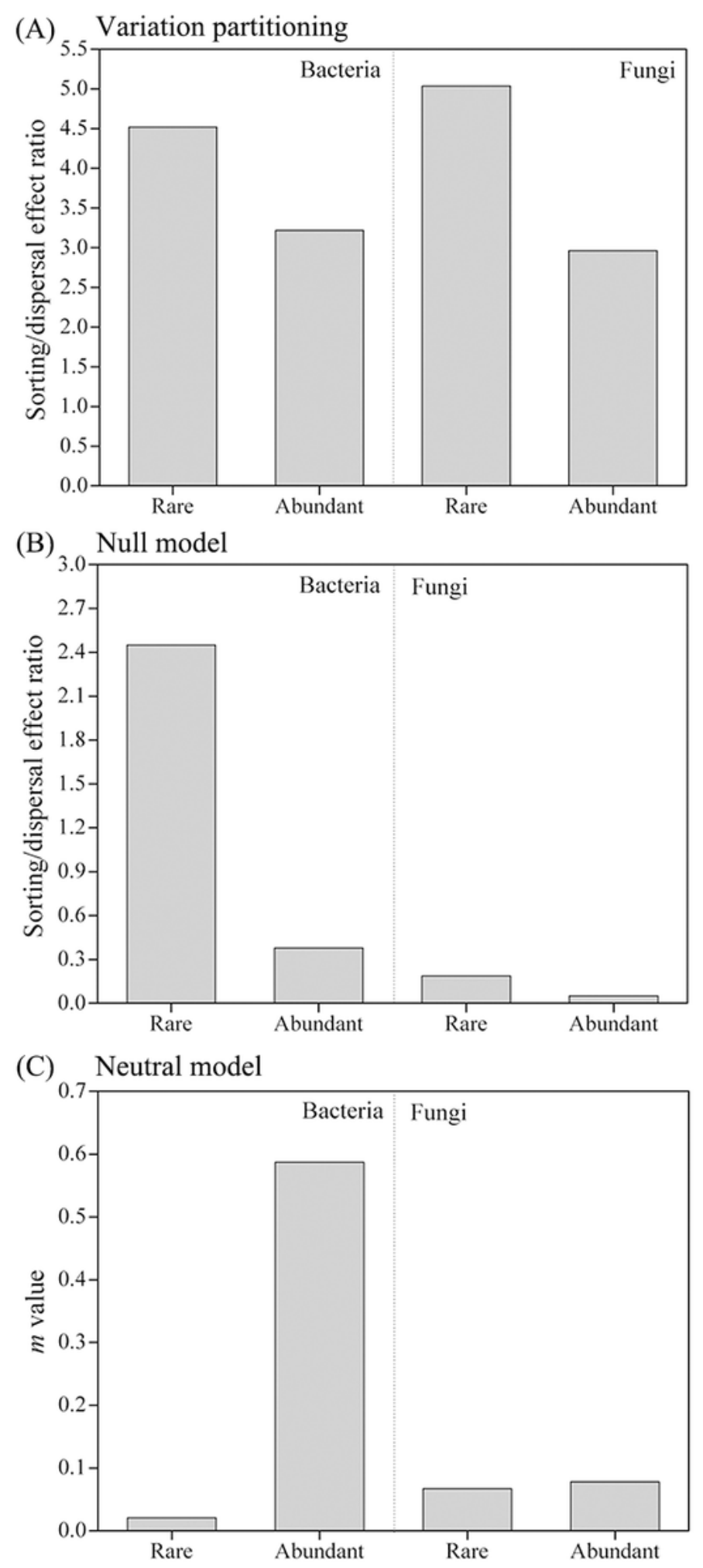

\section{Figure 5}

Community assembly patterns of rare and abundant microbial taxa using variation partitioning $(A)$, null model (B), and neutral model (C) 


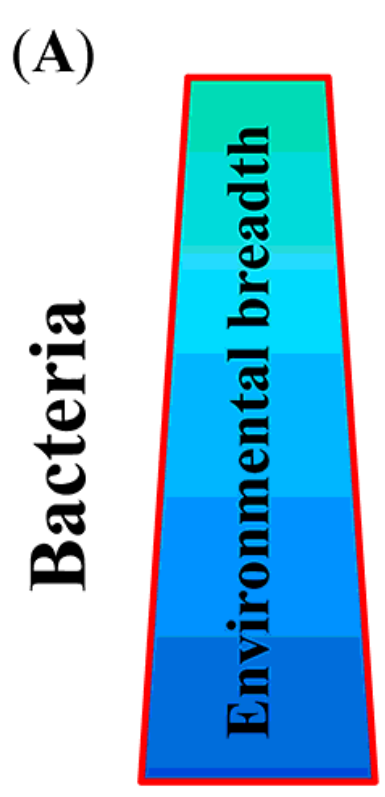

\section{Rare}

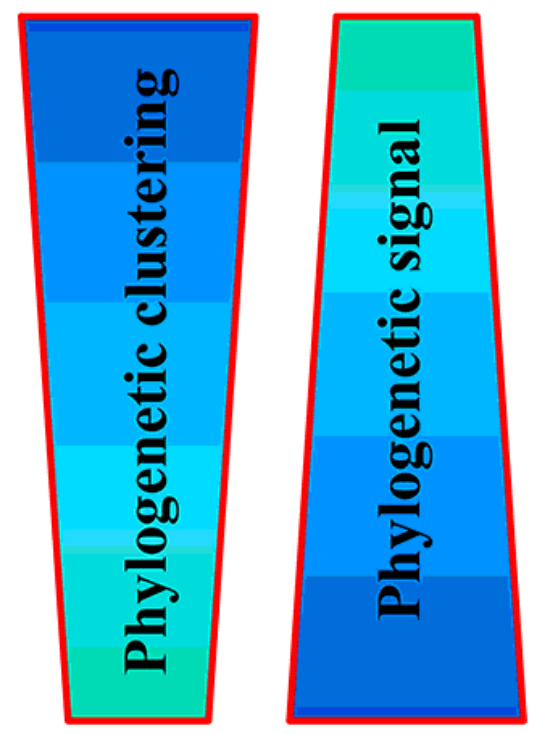

\section{Abundant}

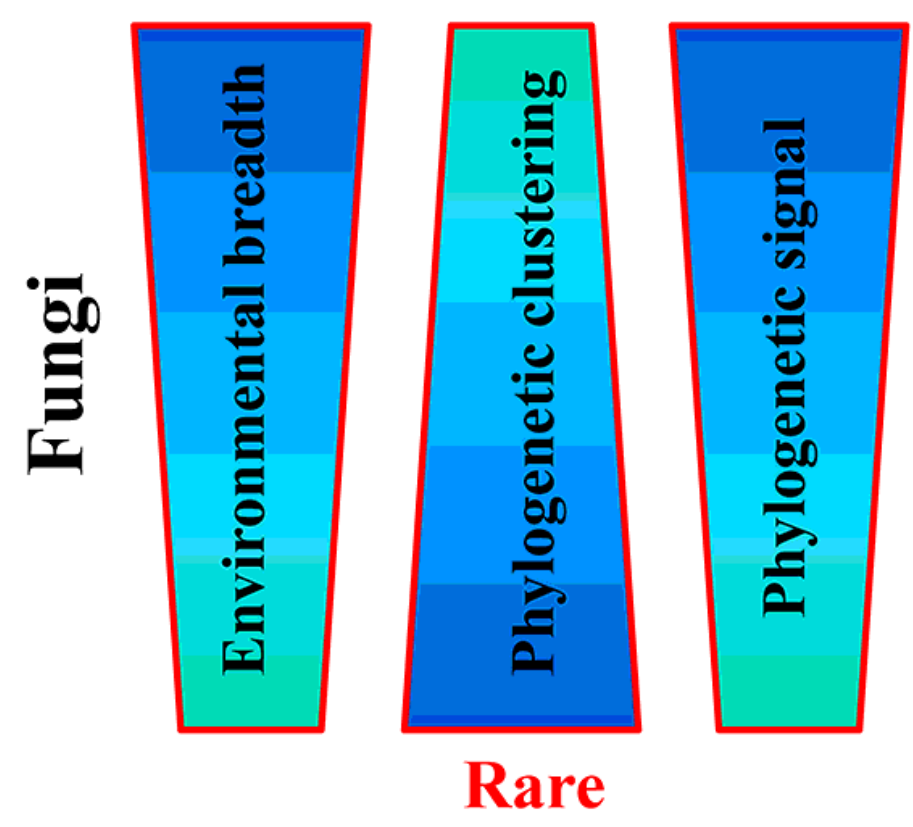

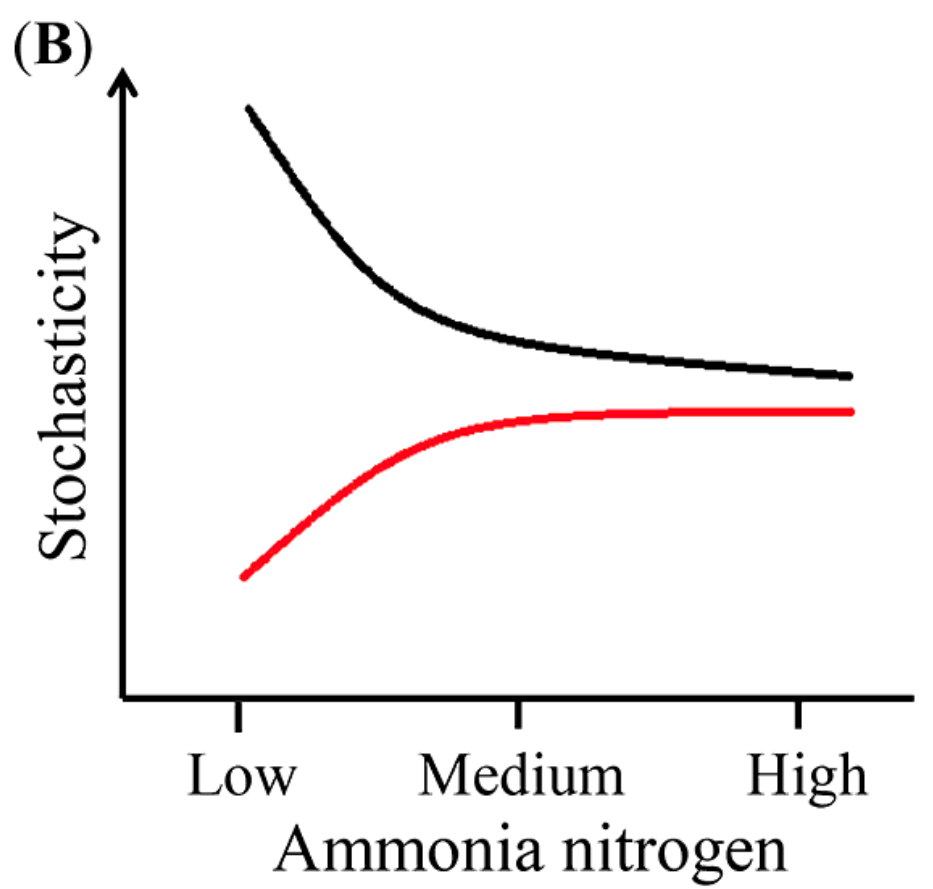

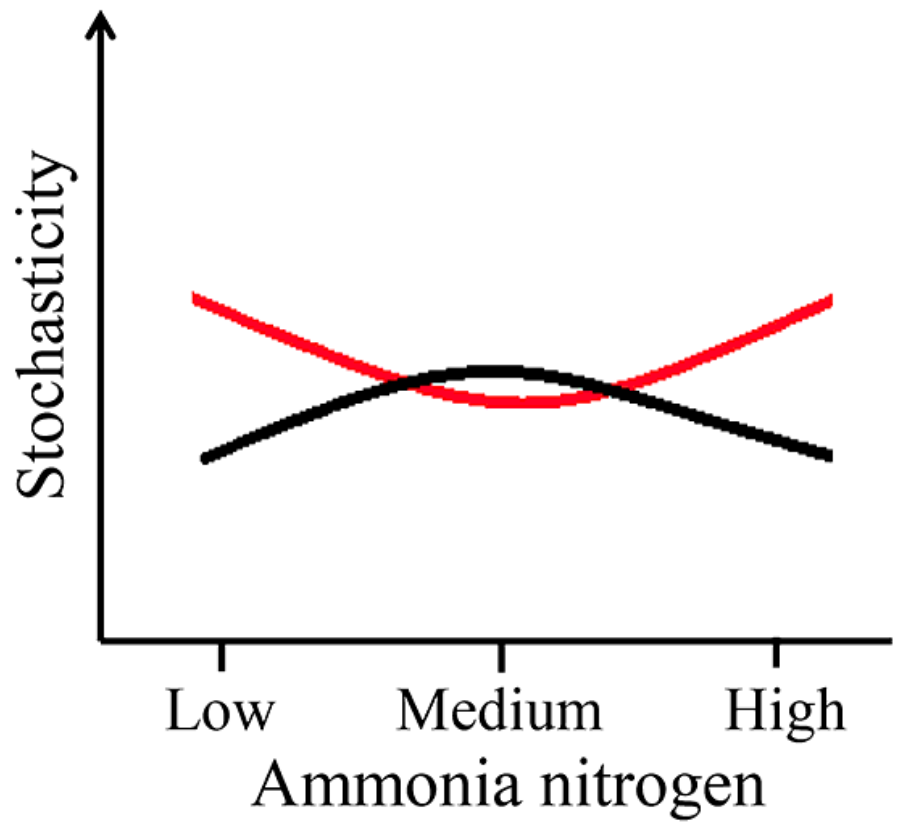

Figure 6

Conceptual models revealing environmental adaptation (A) and stochastic processes (B) in the assembly of rare and abundant microbial sub-communities under the influence of soil ammonia nitrogen

\section{Supplementary Files}

This is a list of supplementary files associated with this preprint. Click to download.

- Additionalfile1.docx 